\title{
MTA1, a metastasis-associated protein, in endothelial cells is an essential molecule for angiogenesis
}

\author{
MIZUHO ISHIKAWA $^{1}$, MITSUHIKO OSAKI ${ }^{1,2}$, NARUMI UNO ${ }^{2,3}$, \\ TAKAHITO OHIRA ${ }^{2,3}$, HIROYUKI KUGOH ${ }^{2,3}$ and FUTOSHI OKADA ${ }^{1,2}$ \\ ${ }^{1}$ Division of Experimental Pathology, ${ }^{2}$ Chromosome Engineering Research Center, \\ ${ }^{3}$ Division of Genome and Cellular Functions, Faculty of Medicine, Tottori University, Yonago, Tottori 683-8503, Japan
}

Received August 5, 2021; Accepted October 14, 2021

DOI: $10.3892 / \mathrm{mmr} .2021 .12527$

\begin{abstract}
Our previous study revealed that metastasis-associated protein 1 (MTA1), which is expressed in vascular endothelial cells, acts as a tube formation promoting factor. The present study aimed to clarify the importance of MTA1 expression in tube formation using MTA1-knockout (KO) endothelial cells (MTA1-KO MSS31 cells). Tube formation was significantly suppressed in MTA1-KO MSS31 cells, whereas MTA1-overexpression MTA1-KO MSS31 cells regained the ability to form tube-like structures. In addition, western blotting analysis revealed that MTA1-KO MSS31 cells showed significantly higher levels of phosphorylation of non-muscle myosin heavy chain IIa, which resulted in suppression of tube formation. This effect was attributed to a decrease of MTA1/S100 calcium-binding protein A4 complex formation. Moreover, inhibition of tube formation in MTA1-KO MSS31 cells could not be rescued by stimulation with vascular endothelial growth factor (VEGF). These results demonstrated that MTA1 may serve as an essential molecule for angiogenesis in endothelial cells and be involved in different steps of the angiogenic process compared with the VEGF/VEGF receptor 2 pathway. The findings showed that endothelial MTAl and its pathway may serve as promising targets for inhibiting tumor angiogenesis, further supporting the development of MTA1-based antiangiogenic therapies.
\end{abstract}

\section{Introduction}

Metastasis-associated protein 1 (MTA1), a member of the MTA family, was first identified by Toh et al (1). MTA1 expression is reported to be associated with tumor malignancy in several cancer types, including esophageal, gastrointestinal,

Correspondence to: Dr Mitsuhiko Osaki, Division of Experimental Pathology, Faculty of Medicine, Tottori University, 86 Nishi-cho, Yonago, Tottori 683-8503, Japan

E-mail: osamitsu@tottori-u.ac.jp

Key words: angiogenesis, endothelial cells, metastasis-associated protein 1, vascular endothelial growth factor, tumor non-small-cell lung, breast and ovarian cancer (2-6). In addition, MTA1 promotes tumor cell proliferation and invasion, and tumor angiogenesis and metastasis (7-9).

However, the precise functional role of endothelial MTAI in angiogenesis remains unclear. Our previous study examined the function of MTA1 in endothelial cells by MTA1 knockdown and found that MTAl small interfering (si)RNA significantly inhibited tube formation but not the proliferation and migration of endothelial cells (10). In addition, it was demonstrated that MTAl knockdown induced a decrease in S100 calcium-binding protein A4 (S100A4) expression and an increase in phosphorylated non-muscle myosin heavy chain IIa (NMIIa). This phosphorylation level of NMIIa may influence the formation of tube structures via altered cytoskeletal dynamics in endothelial cells (10). To evaluate the function of MTAl further, it is necessary to confirm the inhibition of tube formation using MTAl-knockout (KO) cells and to evaluate whether the ability of tube formation is restored by re-expressing MTA1 in the KO cells.

In the present study, MTA1-KO endothelial cells (MTA1-KO MSS31 cells) were established and examined to clarify the role of MTA1 expression in tube formation by endothelial cells. The relationship between the inhibition of tube formation in MTA1-KO MSS31 cells and the vascular endothelial growth factor (VEGF)/VEGF receptor 2 (VEGFR2) pathway was also investigated.

\section{Materials and methods}

Cell culture and reagents. Mouse endothelial cells (MSS31 cells), a gift from Dr H Endo (The University of Tokyo, Tokyo, Japan), and MTA1-KO MSS31 cells were maintained in Dulbecco's modified Eagle's medium (DMEM)/Ham's F-12 medium (Nissui Pharmaceuticals Co., Ltd.) supplemented with $10 \%$ fetal bovine serum (FBS; Sigma-Aldrich; Merck KGaA) at $37^{\circ} \mathrm{C}$ in a humidified $5 \% \mathrm{CO}_{2} / 95 \%$ air mixture. MSS31 cells and MTA1-KO MSS31 cells $\left(3 \times 10^{5}\right)$ were transfected with $2 \mu \mathrm{g}$ MTA1 expression vector or empty vector as a negative control provided by Dr K Takenaga (Chiba Cancer Center Research Institute, Chiba, Japan) using Lipofectamine ${ }^{\circledR} 2000$ (Invitrogen; Thermo Fisher Scientific, Inc.) according to the manufacturer's protocol at room temperature for $20 \mathrm{~min}$. The transfected cells were cultured for $24 \mathrm{~h}$ and used in subsequent 
experiments. When conducting subsequent experiments, each experiment had a non-treatment group.

Engineering of guide ( $g$ )RNA and CRISPR-Cas9 vector for $M T A 1 \mathrm{KO}$. The gRNA for MTAl KO targeting the first exon of MTAl on the coding strand was engineered using the online CRISPR design tool (version 1.3) (http://crispr.mit.edu/). The targeted sequence was 5'-CAGGATTGAAGAGCTTAACA AGG-3'. Complementary guide oligonucleotides (forward, 5'-CACCGCAGGATTGAAGACCTTAACA-3' and reverse, 5'-AAACTGTTAAGCTCTTCAATCCTGC-3') were custom synthesized separately by Sigma-Aldrich (Merck KGaA), annealed and cloned into the $B b s$ I site of pSpCas9(BB)-2A-GFP (pX458). pX458 was a gift from Feng Zhang (Addgene; cat. no. 48138; http://n2t.net/addgene:48138; Research Resource Identifiers (RRID; Addgene_48138) (11).

Electroporation and cell sorting. MSS31 cells were harvested by trypsinization using $0.1 \%$ trypsin and counted using a hemacytometer. Thereafter, $1-2 \times 10^{6}$ cells were resuspended with $3 \mu \mathrm{g}$ plasmid DNA in $100 \mathrm{ml}$ electroporation buffer, transferred to a $0.2 \mathrm{~cm}$ cuvette (Nepa Gene Co., Ltd.) and electroporated using a square electric pulse generating electroporator (NEPA21; Nepa Gene Co., Ltd.) with poring pulse (voltage, $150 \mathrm{~V}$; pulse length, $7.5 \mathrm{msec}$; pulse interval, $50 \mathrm{msec}$; number of pulses, 2; decay rate, $10 \%$; polarity, +) and transfer pulse (voltage, $20 \mathrm{~V}$; pulse length, $50 \mathrm{msec}$; pulse interval, $50 \mathrm{msec}$; number of pulses, 5; polarity, +/-). The cells were transferred to DMEM/Ham's F-12 medium (Nissui Pharmaceuticals Co., Ltd.) supplemented with 10\% FBS (Sigma-Aldrich; Merck KGaA) and seeded in $60 \mathrm{~mm}$ dishes. After $24 \mathrm{~h}$ of electroporation, the transfected populations were sorted by GFP expression using MoFlo XDP cell sorter, equipped with $488 \mathrm{~nm}$ blue laser (Beckman Coulter, Inc.). GFP was excited by $488 \mathrm{~nm}$ blue laser and its emission detected using a 529/28 Band pass (BP) filter. Single cells were sorted into 384-well microplates and cultured for $>1$ month to allow for colony formation.

Genomic DNA isolation, PCR and cloning for DNA sequencing. Genomic DNA was extracted from cells using the Gentra Puregene Cell kit (Qiagen $\mathrm{GmbH}$ ) according to the manufacturer's instructions and PCR analyses were performed as follows. The PCR amplification of the targeted regions was performed with TaKaRa Ex Taq Hot Start (Takara Bio, Inc.) using PCR gene-specific forward (5'-CTCTCTGGGCTCTG TCCATC-3') and reverse (5'-CGGACCCACTCTCAGTC TCT-3') primers. The following temperature protocol was used for PCR: $98^{\circ} \mathrm{C}$ for $1 \mathrm{~min}$; 40 cycles of $98^{\circ} \mathrm{C}$ for $15 \mathrm{sec}, 60^{\circ} \mathrm{C}$ for $15 \mathrm{sec}$ and $72^{\circ} \mathrm{C}$ for $40 \mathrm{sec}$; and $72^{\circ} \mathrm{C}$ for $7 \mathrm{~min}$. The PCR reaction mixture contained each $0.25 \mu \mathrm{M}$ forward and reverse primer, 1.25 units Ex Taq HS polymerase (Takara Bio, Inc.), 1X Ex Taq Buffer (Takara Bio, Inc.), $0.2 \mathrm{mM}$ each dNTP mixture (Takara Bio, Inc.) and 50 ng genomic DNA. PCR products were directly sequenced using specific primers and cloned into the $\mathrm{pCR}^{\mathrm{TM}} 4-\mathrm{TOPO}^{\circledR} \mathrm{TA}$ vector (Thermo Fisher Scientific, Inc.) using the $\mathrm{TOPO}^{\mathrm{TM}} \mathrm{TA}$ Cloning ${ }^{\mathrm{TM}} \mathrm{Kit}$ for Sequencing, without competent cells (Thermo Fisher Scientific, Inc.) following the manufacturer's instructions. Plasmid DNA was isolated using the PureYield ${ }^{\mathrm{TM}}$ Plasmid Miniprep System
(Promega Corporation). Plasmids were sequenced using the M13 forward primer (5'-GTAAAACGACGGCCAG-3') and M13 reverse primer (5'-CAGGAAACAGCTATGAC-3').

Tube formation assay. For the tube formation assays, 24-well plates were coated with Geltrex (Thermo Fisher Scientific, Inc.), which was allowed to solidify overnight at $4^{\circ} \mathrm{C}$. MSS31 cells and MTA1-KO MSS31 cells $\left(8 \times 10^{4}\right.$ cells/well) were seeded and cultured in serum-free medium or serum-free medium supplemented with VEGF-A (10 ng/ml; R\&D Systems, Inc.). Tube formation was evaluated after 4,5 or $19 \mathrm{~h}$. Images of two to three random fields for each sample (magnification, $x 4)$ were captured using an all-in-One Fluorescence Microscope BZ-X710 microscope (Keyence Corporation). The number of junctions was quantified using an Angiogenesis Analyzer (developed by Gilles Carpentier) (https://imagej. nih.gov/ij/macros/toolsets/Angiogenesis\%20Analyzer.txt) in ImageJ [version 1.52v (National Institute of Health)]. Each experiment was repeated three or four times.

Western blotting. Cells were washed in cold PBS and lysed in lysis buffer containing $20 \mathrm{mM}$ Tris- $\mathrm{HCl}(\mathrm{pH} 7.4)$, $150 \mathrm{mM} \mathrm{NaCl}, 0.1 \% \mathrm{SDS}, 1 \%$ sodium deoxycholate, $1 \%$ Triton $\mathrm{X}-100,1 \mu \mathrm{g} / \mathrm{ml}$ aprotinin and $1 \mu \mathrm{g} / \mathrm{ml}$ leupeptin. Lysates were centrifuged at $13,000 \mathrm{x}$ g for $5 \mathrm{~min}$ at $4^{\circ} \mathrm{C}$. Protein concentrations were estimated using the Bradford protein assay (Bio-Rad Laboratories, Inc.) with bovine serum albumin (Fujifilm Wako Pure Chemical Co.) as the standard. Proteins were loaded $30 \mu \mathrm{g}$ per lane and resolved by SDS-PAGE using 8, 10, 12 and $15 \%$ gels, and then electrotransferred to PVDF membranes (MilliporeSigma). After being blocked with $5 \%$ fat-free milk for $2 \mathrm{~h}$ at room temperature, the membranes were blotted using primary antibodies overnight at $4^{\circ} \mathrm{C}$, washed using PBS with $1 \%$ Tween-20 and then incubated with secondary antibodies for $20 \mathrm{~min}$ at room temperature. After washing using PBS with $1 \%$ Tween-20, the bound antibodies were detected using ECL Prime Western Blotting Detection Reagent (RPN2236; Cytiva). The primary antibodies used in the present study were: Anti-MTA1 polyclonal antibody (1:2,000; cat. no. ab71153; Abcam), anti-S100A4 polyclonal antibody (1:1,000; cat. no. 07-2274; MilliporeSigma), anti-NMIIa polyclonal antibody (1:1,000; cat. no. 3403; Cell Signaling Technology, Inc.), anti-phosphorylated (p)-NMIIa polyclonal antibody (1:1,000; cat. no. 5026; Cell Signaling Technology, Inc.), anti-VEGFR2 polyclonal antibody (1:1,000; cat. no. 9698; Cell Signaling Technology, Inc.), anti-p-VEGFR2 (Tyr 1175) polyclonal antibody (1:1,000; cat. no. 3770; Cell Signaling Technology, Inc.), anti- $\beta$-actin monoclonal antibody $(1: 2,000$; cat. no. A5441; Sigma-Aldrich; Merck KGaA). The secondary antibodies used in the present study were: Goat anti-mouse IgG-HRP (1:2,000; cat. no. PM009-7; Medical and Biological Laboratories Co., Ltd.) and goat anti-rabbit IgG-HRP (1:2,000; cat. no. sc-2004; Santa Cruz Biotechnology, Inc.). Densitometry was performed using ImageJ (version $1.52 \mathrm{v}$; National Institute of Health). For the western blotting for the phosphorylation of VEGFR2, wild-type MSS31 cells and MTA1-KO clones were treated with VEGF $(10 \mathrm{ng} / \mathrm{ml})$ for $30 \mathrm{~min}$ and were lysed in lysis buffer. For the western blotting for MTA1 expression by VEGF treatment, MSS31 cells 
treated with $\operatorname{VEGF}(0,10,50$ or $100 \mathrm{ng} / \mathrm{ml})$ for 24,48 or $72 \mathrm{~h}$ and were lysed in lysis Buffer. Each experiment was repeated three or four times.

Reverse transcription-quantitative $(R T-q) P C R$. Total RNA was extracted using TRIzol ${ }^{\circledR}$ reagent (Thermo Fisher Scientific, Inc.) and $1 \mu \mathrm{g}$ total RNA was reverse transcribed into cDNA using the TaKaRa PrimeScript RT master mix (Takara Bio, Inc.) according to the manufacturer's protocol. Subsequently, $2 \mu \mathrm{l}$ cDNA was used for quantitative PCR. qPCR was performed on a Roche LightCycler 480 (Roche Diagnostics) using SYBR Premix Ex Taq II (Tli RNaseH Plus; Takara Bio, Inc.). The primer sequences are as follows: MTAI forward, 5'-GCGGCGAATGAACTGGA-3' and reverse, 5'-TTGGTTTCTGAGGATGAGAGCA-3'; and $\beta$-actin forward, 5'-AGAGGGAAATCGTGCGTGAC-3' and reverse, 5'-CAATAGTGATGACCTGGCCGT-3'. The following thermocycling conditions were used for qPCR: $95^{\circ} \mathrm{C}$ for $30 \mathrm{sec}$; $95^{\circ} \mathrm{C}$ for $10 \mathrm{sec}$; followed by 45 cycles of $95^{\circ} \mathrm{C}$ for $10 \mathrm{sec}$ and $60^{\circ} \mathrm{C}$ for $1 \mathrm{~min}$. $\beta$-actin was used as the internal control. The results are expressed as the fold change between the expression level of each mRNA and the internal reference using the $2^{-\Delta \Delta \mathrm{Cq}}$ method (12). Each experiment was repeated three times.

Immunoprecipitation. Cells were washed in PBS and lysed in lysis buffer A [25 mM Tris- $\mathrm{HCl}(\mathrm{pH} 7.5), 420 \mathrm{mM} \mathrm{NaCl}$, $0.5 \%$ Triton $\mathrm{X}-100,5 \mathrm{mM} \mathrm{CaCl}_{2}, 10 \mu \mathrm{g} / \mathrm{ml}$ aprotinin, $10 \mu \mathrm{g} / \mathrm{ml}$ leupeptin and $1 \mathrm{mM}$ phenylmethylsulfonyl fluoride (PMSF)] for $30 \mathrm{~min}$ at $4^{\circ} \mathrm{C}$. Lysates were diluted 1.8 -fold with lysis buffer B [25 mM Tris-HCl (pH 7.5), 0.5\% Triton X-100, $5 \mathrm{mM} \mathrm{CaCl}, 10 \mu \mathrm{g} / \mathrm{ml}$ aprotinin, $10 \mu \mathrm{g} / \mathrm{ml}$ leupeptin and $1 \mathrm{mM}$ PMSF] and were then centrifuged at $13,000 \mathrm{x} \mathrm{g}$ for $10 \mathrm{~min}$ at $4^{\circ} \mathrm{C}$. Immunoprecipitation assays were performed using $50 \mu \mathrm{l}$ of the non-magnetic sepharose beads (nProtein A Sepharose 4 Fast Flow or nProtein G Sepharose 4 Fast Flow; (cat. no. 17528001, 17061801; Cytiva) according to the manufacturer's instructions. Briefly, $500 \mu \mathrm{l}$ of the supernatants containing proteins was incubated for $1 \mathrm{~h}$ at $4^{\circ} \mathrm{C}$ with $3 \mu \mathrm{g}$ anti-MTA1 polyclonal antibody (cat. no. ab71153; Abcam), anti-S100A4 monoclonal antibody (cat. no. CPTC-S100A4-3; Developmental Studies Hybridoma Bank), normal rabbit IgG (cat. no. 2729; Cell Signaling Technology, Inc.) or normal mouse IgG (I-8765; Sigma-Aldrich; Merck KGaA). Immune complexes were recovered on nProtein A Sepharose beads or nProtein $\mathrm{G}$ Sepharose beads for $1 \mathrm{~h}$ at $4^{\circ} \mathrm{C}$. The immunoprecipitants were washed five times with TBS $[50 \mathrm{mM}$ Tris- $\mathrm{HCl}$ ( $\mathrm{pH} 7.5), 150 \mathrm{mM} \mathrm{NaCl}$ and then $2 \mathrm{X}$ additional buffer [1 $\mathrm{M}$ Tris- $\mathrm{HCl}$ (pH 6.8), 10\% SDS, 100\% glycerol, bromophenol blue] was added. After boiling for $3 \mathrm{~min}$ at $95^{\circ} \mathrm{C}$, the supernatants were used as western blotting samples. Western blotting was according to the aforementioned protocol. The experiment was repeated four times.

Statistical analysis. The number of samples to be analyzed was predetermined based on the requirement of the statistical test used. Statistical analysis was performed using a personal computer with the program Excel Statistics version 3.0 (Esumi Co. Ltd.) functioning on the program Excel (Microsoft Corporation). Data are shown as the mean \pm standard deviation. Comparisons between two groups were analyzed using the unpaired Student's t-test. Comparisons among multiple groups were analyzed using one-way ANOVA followed by Dunnett's post hoc test. The researchers involved were not blinded and the samples were not randomized during sample collection or data analysis. $\mathrm{P}<0.05$ was considered to indicate a statistically significant difference.

\section{Results}

Engineering of MTA1-KO MSS31 clones. To gain deeper insight into the functions of $M T A 1$, the present study designed gRNA targeting MTA1 and used the CRISPR-Cas9 system to generate MTA1-KO MSS31 cell lines. A total of two colonies (\#1 and \#2) grown from individual cells were sorted via FACS. These colonies were confirmed as MTA1 KO by RT-qPCR (data not shown) and western blotting (Fig. 1A). The deletion of MTA1 in the cellular genome of the two colonies was also checked by genome sequencing (Fig. 1B). Clone \#1 had a four-base deletion in one allele and a 116-base deletion. Clone \#2 had a five-base deletion in one allele and a 201-base deletion. A frameshift mutation due to multiple-base deletion in each allele resulted in the loss of wild-type MTA1 protein expression. These results indicated the successful generation of MTA1-KO MSS31 cell clones.

MTAl expression contributes to tube formation in vitro. Previously, we reported that suppression of MTA1 in endothelial cells inhibited tube formation in vitro (10). The present study performed a tube formation assay using wild-type MSS31 cells and two MTA1-KO clones to determine the effect of tube formation in MTA1-KO clones. Images were captured of MSS31 cells and two MTA1-KO clones at $5 \mathrm{~h}$ after the cells were plated onto Geltrex (Fig. 2A) and analyzed using the Angiogenesis Analyzer. An example of the computer-adjusted image to evaluate tube formation and junction is shown in Fig. 2B. At $5 \mathrm{~h}$ after the cells were seeded, wild-type MSS31 cells showed tube formation spanning the entire well. Compared with the wild-type MSS31 cells, the two MTA1-KO clones showed significantly impaired tube formation (Fig. 2A and C).

It was also hypothesized that diminished tube formation in MTA1-KO clones could be recovered by MTA1 overexpression. After MTA1 overexpression in MTA1-KO MSS31 clones transfected with MTA1 expression vector [MTA1-KO + overexpression (OE) MSS31 clones] was confirmed by RT-qPCR and western blotting (Fig. S1A and B), a tube formation assay was performed using MTA1-KO clones and MTA1-KO + OE MSS31 clones. At $5 \mathrm{~h}$ after the cells were seeded, tube formation was not observed in the MTA1-KO + OE MSS31 clones (Fig. S2). At $19 \mathrm{~h}$ after the cells were plated onto Geltrex, short and incomplete tube-like structures were observed in MTA1-KO + OE MSS31 clones; however, no tube formation was observed in MTA1-KO clones that were transfected with empty vector or remained untransfected (Fig. 3A and B). These results indicated that the inhibition of tube formation caused by MTAl KO was recovered by MTA1 overexpression.

Involvement of MTA1/S100A4 interaction and p-NMIIa in tube formation in MTA1-KO and MTA1-KO + OE MSS31 cells. We previously reported the mechanism of tube formation 
A

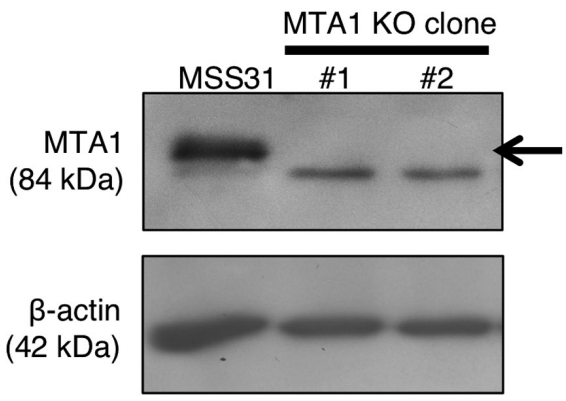

B

$\begin{array}{rr}\text { MSS31 } & \text { CTGGGTATTGAACTGAGCCATCTCTCCAGCCCCTTTGGGGGGGTGGGGTGGGCTGTCTT } \\ \text { MTA1KO clone \#1-allele } 1 & \text { cTGGGTATGAACTGAGCCATCTCTCCAGCCCCTTGGGGCGGTGGGGGGGGCTGTCTT } \\ \text { MTA1KO clone \#1-allele } 2 & \text { cTGGGTATTGAACTGAGCCATCTCTCCAGCCCCTTTGGGGGGGGGGGGGGCTGTCTT } \\ \text { MTA1KO clone \#2-allele } 1 & \text { cTGGGTATTGAACTGAGCCATCTCTCCAGCCCCTTGGGGGGGGGGGGGGCTGTCT } \\ \text { MTA1KO clone \#2-allele } 2 & \text { cTGGGTATTGAACTGAGCCATCTCTCCAGCCCCTTTGGGGGGTGGGGGGG------- }\end{array}$

MSS31 AAATGGTGGCAATGACTTAGAGACCTGGTGCTATGTGTTTATCCAGATTTCTCTGTTTGC MTA1KO clone \#1-allele 1 AAATGGTGGCAATGACTTAGAGACCTGGTGCTATGTGTTTATCCAGATTTCTCTGTTTGC MTA1KO clone \#1-allele 2 AAATGGTGGCAATGACTTAGAGACCTGGTGCTATGTGTTTATCCAGATTTCTCTGTTTGC MTA1KO clone \#2-allele 1 AAATGGTGGCAATGACTTAGAGACCTGGTGCTATGTGTTTATdCAGATTTCTCTGTTTGC MTA1KO clone \#2-allele 2 gRNA

MSS31 AGACTATGTCTACTTTGAGAACTCCTCCAGCAACCCGTACCTGATCCGCAGGATTGAAGA MTA1KO clone \#1-allele 1 AGACTATGTCTACTTTGAGAACTCCTCCAGCAACCCGTACCTGATCCGCAGGATTGAAGA

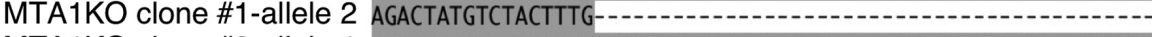
MTA1KO clone \#2-allele 1 AGACTATGTCTACTTTGAGAACTCCTCCAGCAACCCGTACCTGATCCGCAGGATTGAAGA MTA1KO clone \#2-allele 2

MSS31 $\underset{\text { GCTTAACAAGGTGAGAGCTCAACACTGCTGCCTGCACCTCCCCGCCCCCCGCCCGTGGAG }}{\longrightarrow}$ MTA1KO clone \#1-allele 1 GCTT----AGGTGAGAGCTCAACACTGCTGCCTGCACCTCCCCGCCCCCCCGCCCGTGGAG MTA1KO clone \#1-allele 2 MTA1KO clone \#2-allele 1 GCTTAA-----TGAGAGCTCAACACTGCTGCCTGCACCTCCCCGCCCCCCGCCCGTGGAG MTA1KO clone \#2-allele 2

MSS31 AGGCTCGATTGGGCCTGCCCTGTTGTGGGTGGTCAGGGTCTTTCTGCCTGGATTTGGATG MTA1KO clone \#1-allele 1 AGGCTCGATTGGGCCTGCCCTGTTGTGGGTGGTCAGGGTCTTTCTGCCTGGATTTGGATG MTA1KO clone \#1-allele 2 --------CCTGCCCTGTTGTGGGGGTCAGGGTCTTTCTGCCTGGATTTGGATG MTA1KO clone \#2-allele 1 AGGCTCGATTGGGCCTGCCCTGTTGTGGGTGGTCAGGGTCTTTCTGCCTGGATTTGGATG MTA1KO clone \#2-allele 2 ---------CCTGCCCTGTTGTGGGGGTCAGGGTCTTTCTGCCTGGATTTGGATG

Figure 1. Generation of MTA1-knockout MSS31 cell lines (MTA1-KO MSS31 cell lines) using the CRISPR/Cas9 system. (A) Western blotting of MTA1 protein expression levels in two MTA1-KO clones compared with those in wild-type MSS31 cells $(n=3)$. The arrow represents the disappearance of MTA1 expression by MTA1 knockout. (B) Alignment of the genomic region including the target sequence of gRNA in the genomic DNA of wild-type MSS31 cells, MTA1-KO clone \#1 and MTA1-KO clone \#2. The arrow represents the gRNA binding site. The first ATG codon is surrounded by a rectangle in the sequence. MTA1, metastasis-associated protein 1; KO, knockout; gRNA, guide RNA.

by MTA1, wherein MTA1 interacts with S100A4 and changes the phosphorylation state of NMIIa (10). Therefore, the present study examined whether inhibition of tube formation by MTAl $\mathrm{KO}$ and its restoration by MTA1 overexpression was associated with the MTA1/S100A4 interaction and the phosphorylation state of NMIIa. First, the MTA1/S100A4 interaction was found to have disappeared in MTA1-KO MSS31 cells, but was detected in MTA1-KO + OE MSS31 cells, as determined via immunoprecipitation using anti-MTA1 or anti-S100A4 antibodies (Fig. 4A and B). Moreover, the phosphorylation of NMIIa was found to be increased by MTA1 KO compared with wild-type MSS31 cells (Fig. 5A and B). The increased NMIIa phosphorylation in MTA1-KO clones was slightly inhibited by MTA1 overexpression; however, the difference was not statistically significant (Fig. 5A and B). Thus, it was proposed that the altered tube formation abilities of MTA1-KO and -OE cells were caused by MTA1/S100A4 interaction and NMIIa phosphorylation.
Relationship between MTA1 and VEGF in MTA1-KO MSS31 cells. Following our previous report that MTAl knockdown suppressed tube formation (10), the present study revealed that MTA1 knockout also inhibited tube formation in vitro. Angiogenesis is influenced by numerous angiogenic pathways, including the VEGF-VEGFR2 pathway (13-15). Therefore, the present study examined whether the suppressed tube formation in MTA1-KO MSS31 cells could be rescued by stimulation with VEGF. It was first confirmed that VEGFR2 was phosphorylated following VEGF stimulation in both wild-type MSS31 cells and MTA1-KO MSS31 cells (Fig. S3A). In addition, VEGFR2 expression levels were not notably altered by MTAl KO. Tube formation was promoted in wild-type MSS31 cells treated with VEGF compared with untreated cells (Figs. 6A, B and S3A). By contrast, despite the phosphorylation of VEGFR2 by VEGF treatment, tube formation could not be rescued in MTA1-KO MSS31 cells treated with VEGF (Figs. 6A, B and S3A). 
A

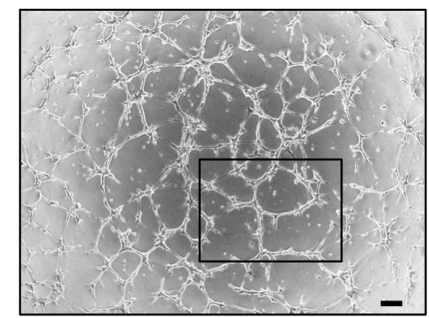

B

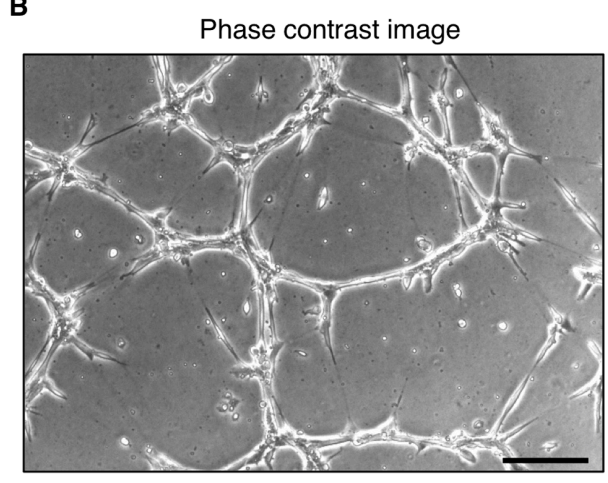

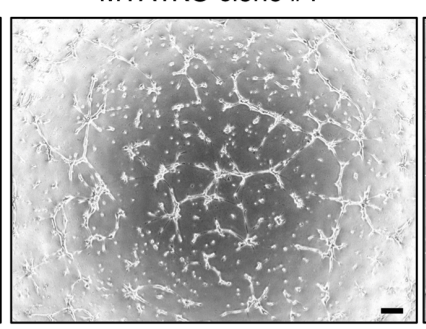

Phase contrast image analyzed by Angiogenesis Analyzer

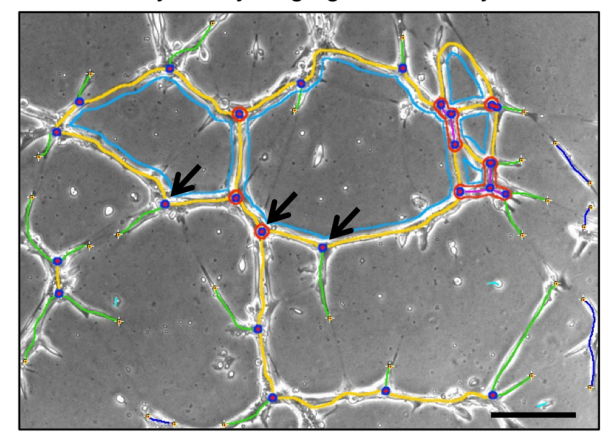

C

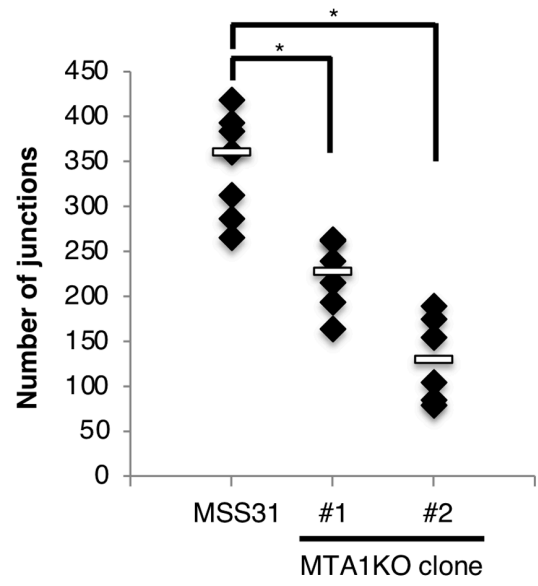

Figure 2. MTA1-KO clones showed inhibition of tube formation at $5 \mathrm{~h}$ after seeding wild-type MSS31 cells and two MTA1-KO clones. (A) Wild-type MSS31 cells and two MTA1-KO clones were seeded onto Geltrex and tube formation was photographed after $5 \mathrm{~h}$. Scale bar, $200 \mu \mathrm{m}$. (B) Enlarged phase contrast image of the area contained within the black box in 2A (left panel). An example of a phase contrast image with the superposition of vectorial objects obtained using the Angiogenesis Analyzer for ImageJ. The arrows represent junctions as shown in red surrounded by blue (right panel). Scale bar, $200 \mu \mathrm{m}$. (C) Quantification of the number of junctions in wild-type MSS31 cells and MTA1-KO clones. Wild-type MSS31 cells, n=7; MTA1-KO clones, n=6. "P<0.05. MTA1, metastasis-associated protein 1; KO, knockout.

In addition, MTA1 expression was not affected by VEGF stimulation (Fig. S3B). These results indicated that VEGF stimulation could not promote tube formation in MTA1-KO MSS31 cells, implying that the role of MTA1 in tube formation may be independent of VEGF.

\section{Discussion}

MTA1 expression in tumor cells is reported to exert multiple functions associated with cancer progression, including proliferation, migration, invasion and tumor angiogenesis (7-9). However, the role of MTA1 in endothelial cells remains to be elucidated. Our group previously reported that MTA1 is involved in tumor angiogenesis via the MTA1/S100A4/NMIIa axis in endothelial cells (10). Therefore, the present study aimed to confirm the function of endothelial MTAl in angiogenesis using MTA1-KO MSS31 cells generated with the CRISPR-Cas9 system.

The phosphorylation level of NMIIa was decreased in the MTA1-KO + OE MSS31 cells compared with the corresponding phosphorylation level in MTA1-KO MSS31 cells and MTA1-KO cells transfected with an empty vector. Although the protein expression level of MTA1 in MTA1-KO + OE MSS31 cells was lower than that in the wild-type MSS31 cells, the interaction between MTA1 and S100A4 occurred in a similar way to that seen in the wild-type MSS31 cells. These results indicated that low levels of MTA1 were sufficient to alter angiogenesis via the interaction with S100A4. Concerning the association between MTA1, NMIIa and tube formation, MTA1-KO MSS31 cells showed an increase in NMIIa phosphorylation and a decrease in tube formation. However, whether the level of the NMIIa phosphorylation 
A MTA1KO clone \#1
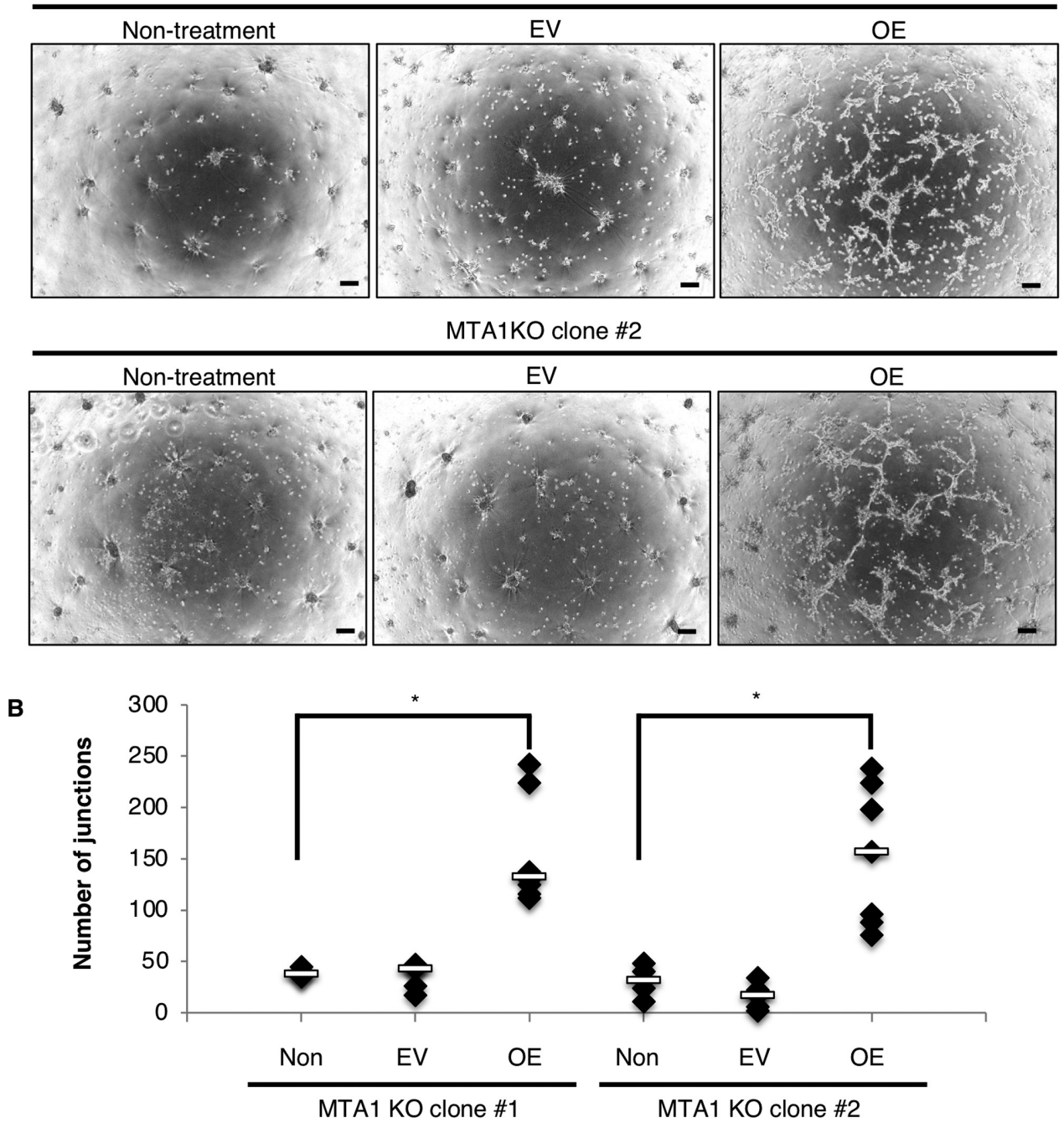

Figure 3. MTA1-KO MSS31 cells transfected with MTA1 expression vector (MTA1-KO + OE MSS31 cells) can partly recover tube formation. (A) Tube formation assays were performed using MTA1-KO MSS31 cells and MTA1-KO + OE MSS31 clones, and images were captured after $19 \mathrm{~h}$. Scale bar, $200 \mu \mathrm{m}$. (B) Quantification of the number of junctions in MTA1-KO MSS31 cells (non-treatment), MTA1-KO cells transfected with EV and MTA1-KO cells transfected with MTA1 expression vector (MTA1-KO + OE MSS31 cells). Non-treatment, $n=6 ; \mathrm{EV}, \mathrm{n}=6 ; \mathrm{OE}, \mathrm{n}=8$. ${ }^{*} \mathrm{P}<0.05$. MTA1, metastasis-associated protein 1; $\mathrm{KO}$, knockout; $\mathrm{OE}$, overexpression; $\mathrm{EV}$, empty vector; non, non-treatment.

directly affected the ability of tube formation was not investigated. Therefore, a mutated form of the phosphorylation site of NMIIa should be generated to investigate this further.

The present study observed that MTA1-KO MSS31 cells displayed impaired tube formation at $5 \mathrm{~h}$ after seeding cells and the tube structure disappeared at $19 \mathrm{~h}$. By contrast, MTA1-KO + OE MSS31 cells partly formed tube-like structures at $19 \mathrm{~h}$, but this was not observed at $5 \mathrm{~h}$, possibly because the direct use of MTA1 expression vector-transfected cells in the tube formation assay caused differences in MTA1 expression levels among the MSS31 cells. These results indicated that endothelial MTAl may serve an important role in tube formation and maintenance.

Angiogenesis involves multiple steps, including protease production, endothelial migration and proliferation, vascular tube formation and blood vessel maturation, that are regulated by numerous angiogenic pathways $(13,16,17)$. A number of signaling pathways are associated with this process, whereby VEGF/VEGFR2 signaling is the most commonly known and serves as an important key pathway $(14,18,19)$. Therefore, the present study examined the association between MTA1 and VEGF in vitro. The tube formation ability was not recovered by treatment of MTA1-KO MSS31 cells with VEGF, although the phosphorylation of VEGFR2 was confirmed in those cells treated with VEGF. These data suggested that the involvement of the MTA1/S100A4/NMIIa pathway in angiogenesis was distinct from the regulation by the VEGF/VEGFR2 signaling pathway. However, the proangiogenic factors and pathways, including the VEGF/VEGFR2 pathway and fibroblast growth factor (FGF)/FGF receptors pathway, primarily control the survival, migration and proliferation of vascular endothelial cells in previous studies $(14,15,18)$.

The present study focused on this difference between the functions of MTA1 and VEGF in the angiogenic process. The angiogenic process can be roughly divided into two steps: Degradation of the basement membrane, proliferation, migration and sprout formation (step 1); and vascular tube formation and maturation (step 2; Fig. 7) $(13,16,17)$. Based on this model, the VEGF/VEGFR2 signaling pathway controls the first step of the angiogenic process. Conversely, the MTA1/S100A4/NMIIa 
A WB : anti-S100A4 antibody

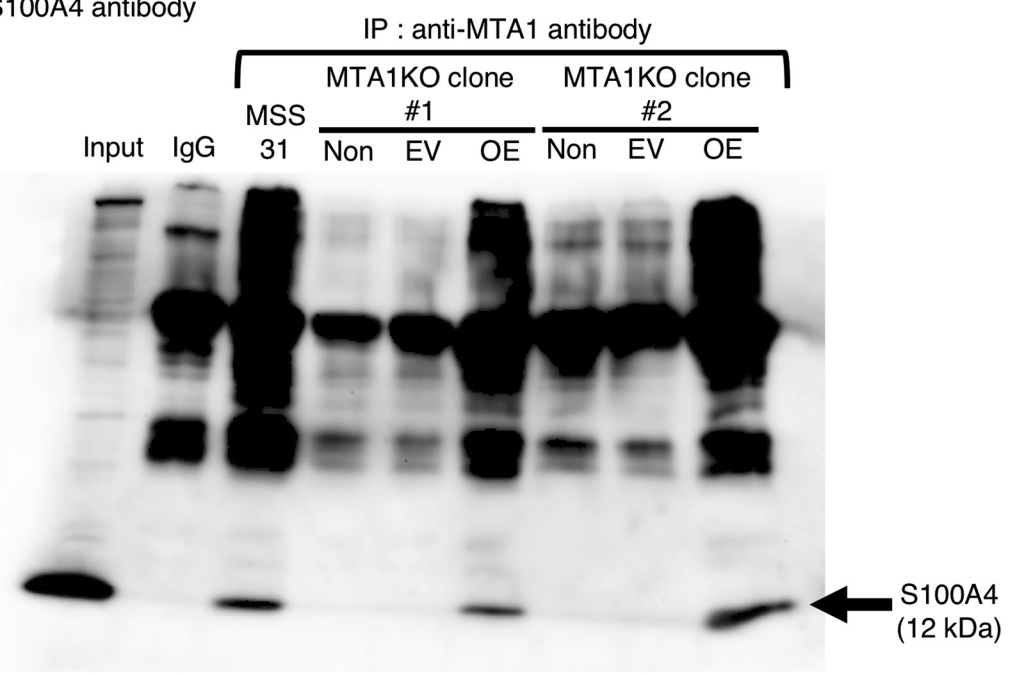

B WB : anti-MTA1 antibody

IP : anti-S100A4 antibody

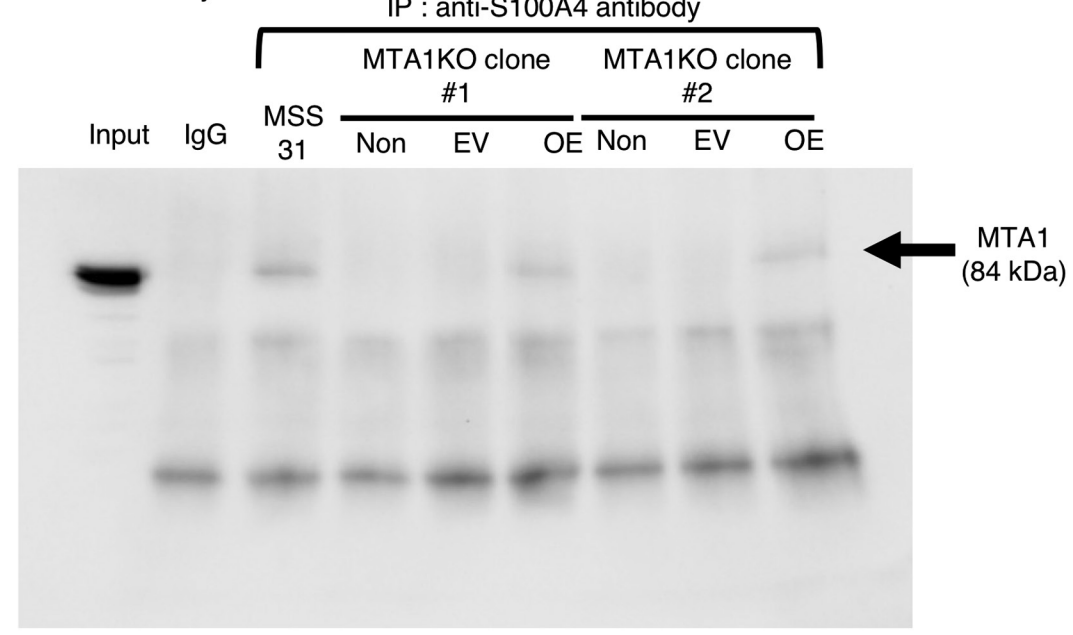

Figure 4. Formation of the MTA1/S100A4 complex in MTA1-KO MSS31 cells and MTA1-KO MSS31 cells transfected with the MTA1 expression vector (MTA1-KO + OE MSS31 cells). (A) WB with anti-S100A4 antibody after IP with the indicated antibodies using wild-type MSS31 cells, MTA1-KO MSS31 cells (non-treatment), MTA1-KO cells transfected with EV and MTA1-KO cells transfected with MTA1 expression vector (MTA1-KO + OE MSS31 cells), Input represents $10 \%$ of the cell lysate. Normal rabbit IgG was used as a negative control (n=3). (B) WB with anti-MTA1 antibody after IP with the indicated antibodies using cell lysates. Normal mouse IgG was used as a negative control $(\mathrm{n}=4)$. MTA1, metastasis-associated protein 1; S100A4, S100 calcium-binding protein A4; KO, knockout; OE, overexpression; IP, immunoprecipitation; EV, empty vector; non, non-treatment; WB, western blotting.

axis primarily regulates tube formation in the second step. In other words, MTA1 and VEGF are involved in the angiogenesis process, but the point at which they exert their functions may be different.

Tumor angiogenesis is an appealing therapeutic target (20). Antiangiogenic therapy has been applied clinically and has emerged as one of the viable treatment options for cancer (21). Due to the pivotal role of the VEGF/VEGFR2 pathway in pathological angiogenesis, most approved antiangiogenic drugs target VEGF-A or its receptors, including VEGFR2 $(22,23)$. Although these treatment strategies have provided substantial clinical benefits in cancer therapeutics, their effects are limited by the development of resistance $(24,25)$. The present study revealed that the MTA1/S100A4 axis in endothelial cells may serve as an important pathway for tube formation and was functionally distinct from the VEGF/VEGFR2 pathway at the site of action in angiogenesis. Thus, therapies targeting the endothelial MTA1/S100A4 axis may provide a novel therapeutic strategy distinct from conventional therapies. In other words, the axis may serve as a useful target molecule for treating patients who manifest VEGF-resistance or show poor responses to VEGF inhibitors because it may be possible to inhibit angiogenesis by blocking step 2 with or without activation of step 1 (Fig. 7). In addition, the combination of antiangiogenic drugs targeting the VEGF/VEGFR2 pathway and the MTA1/S100A4 pathway may induce additive or synergistic effects in the inhibition of tumor angiogenesis and tumor growth, resulting in improved therapeutic outcomes. These possibilities, such as the alternative for the treatment of VEGF-resistance and the enhancement of the antiangiogenic effect using VEGF inhibitors, could be the benefits of targeting the MTA1/S100A4 pathway.

However, there are problems with using MTA1 as a therapeutic target. Since MTA1 forms complexes with other proteins and regulates a number of genes, such as myeloid differentiation factor 88 and cryptochrome $1(26,27)$, suppressing MTA1 itself using MTA1 inhibitors may have negative effects on other pathways. In addition, hypertension 
A

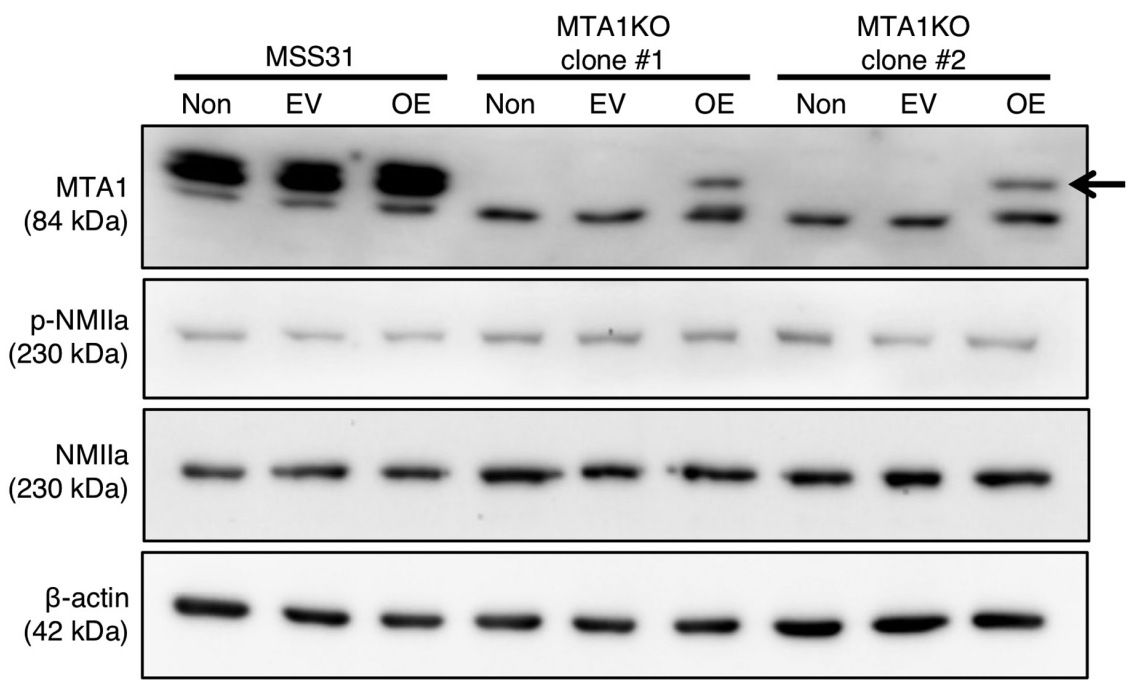

B

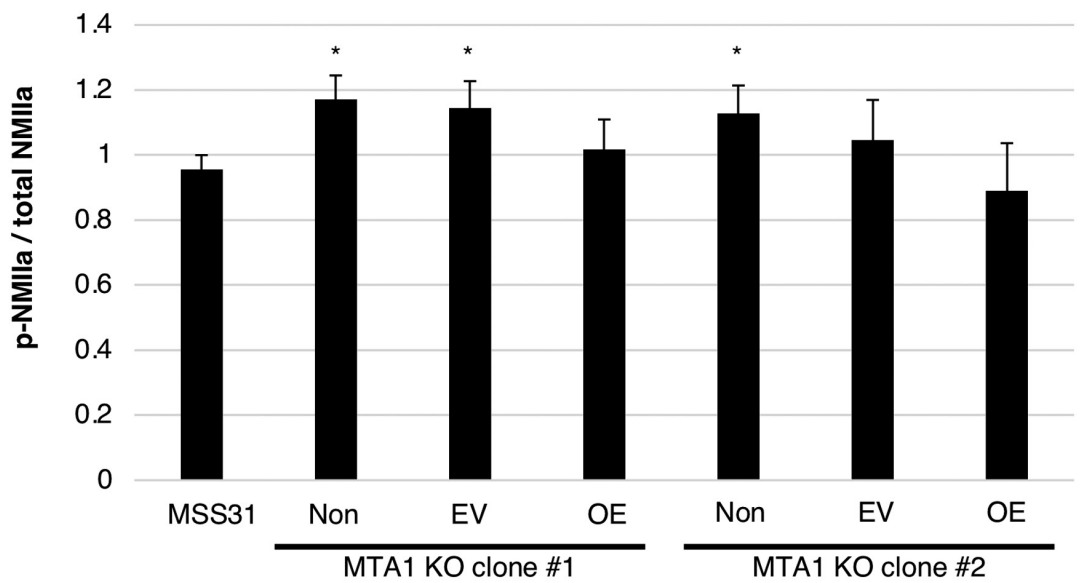

Figure 5. Changes in the phosphorylation status of NMIIa in wild-type MSS31 cells and MTA1-KO MSS31 cells transfected with the MTA1 expression vector. (A) Western blotting of wild-type MSS31 cells, MTA1-KO MSS31 cells (non-treatment), MTA1-KO cells transfected with EV and MTA1-KO cells transfected with MTA1 expression vector (MTA1-KO + OE MSS31 cells) (n=6). (B) p-NMIIa expression levels were measured using ImageJ software and normalized to $\beta$-actin $(\mathrm{n}=6)$. " $\mathrm{P}<0.05$ vs. wild-type MSS31 cells. Data are presented as the mean \pm SD. NMIIa, non-muscle myosin heavy chain IIa; MTA1, metastasis-associated protein 1; KO, knockout; $\mathrm{OE}$, overexpression; EV, empty vector; non, non-treatment; p, phosphorylated.

and impaired wound healing, which are known side effects of antiangiogenic therapy $(14,28)$, may also occur. Therefore, suppressing MTA1 function using molecules that specifically act on the MTA1/S100A4 pathway in antiangiogenic treatment strategies targeting MTA1 is important.

The present study had several limitations. First, the investigation of the MTA1/S100A4 interaction was not complete. The present study reported that this interaction was serial (i.e., the two proteins were associated with each other) as it focused on the formation of the MTA1/S100A4 complex as a mechanism of angiogenesis. However, the possibilities that this interaction is serial, parallel (i.e., the two proteins function independently) or both should be considered. Second, the results are insufficient to definitively conclude whether MTA1 may be involved in angiogenesis regulated by the VEGF/VEGFR2 pathway. The present study demonstrated that the angiogenic process cannot occur normally if MTA1 is absent in vascular endothelial cells even when the cells were stimulated by VEGF. With only this result, the question as to how MTA1 is involved in angiogenesis compared with VEGF cannot be clearly answered. To answer these points, a screening assay should be generated to search for the candidate molecules that inhibit the formation of the MTA1/S100A4 complex. Once the candidate molecules are determined, it is expected that the MTA1/S100A4 interaction and the functions of MTA1 and VEGF in angiogenesis could be shown more clearly by comparing the results of tube formation using MTA1-KO MSS31 cells, MTA1-S100A4 inhibitors and VEGF inhibitors.

Using MTA1-KO MSS31 cells, the present study not only reconfirmed that MTA1 expression in endothelial cells served a crucial role in tube formation and that the mechanism involved the MTA1/S100A4 axis, but also demonstrated that MTA1 was functionally distinct from VEGF in its site of action in angiogenesis. These results were a novel finding and emphasized the importance of MTA1 in angiogenenic process. Based on these findings and the results of our previous study (10), the MTA1/S100A4/NMIIa pathway in endothelial cells may serve as a potential target for suppressing angiogenesis and tumor growth via a mechanism of action different from that of VEGF inhibitors. 


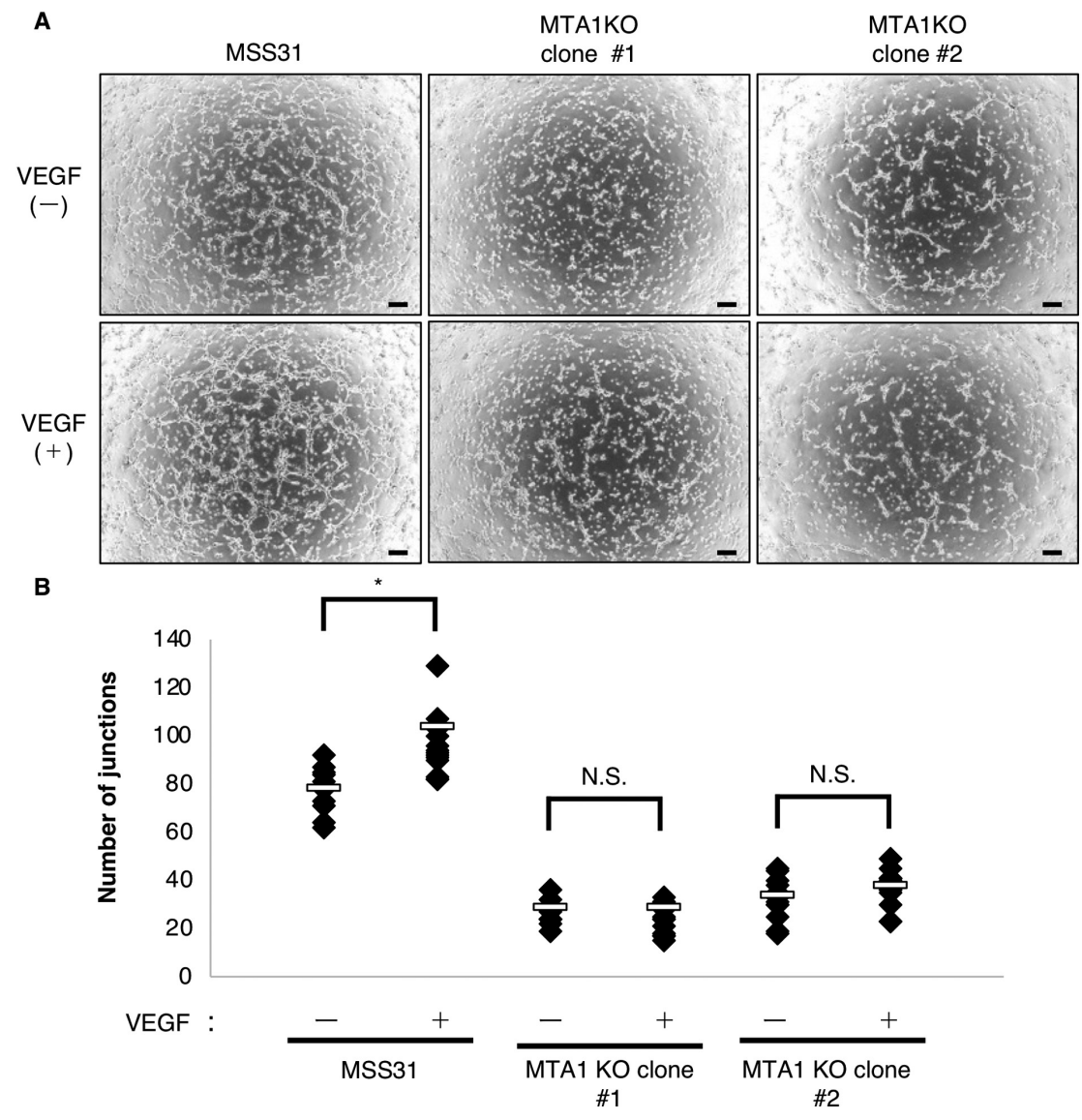

Figure 6. Interaction between MTA1 and VEGF in MTA1-KO MSS31 cells. (A) Tube formation assays were performed using wild-type MSS31 cells and MTA1-KO clones, which were treated with VEGF or untreated. Images were captured after $4 \mathrm{~h}$. Scale bar, $200 \mu \mathrm{m}$. (B) Quantification of the number of junctions in wild-type MSS31 cells and MTA1-KO clones. VEGF(-), $n=16$; VEGF(+), $n=20$. ${ }^{*} \mathrm{P}<0.05$. MTA1, metastasis-associated protein 1; VEGF, vascular endothelial growth factor; KO, knockout; NS, not significant.

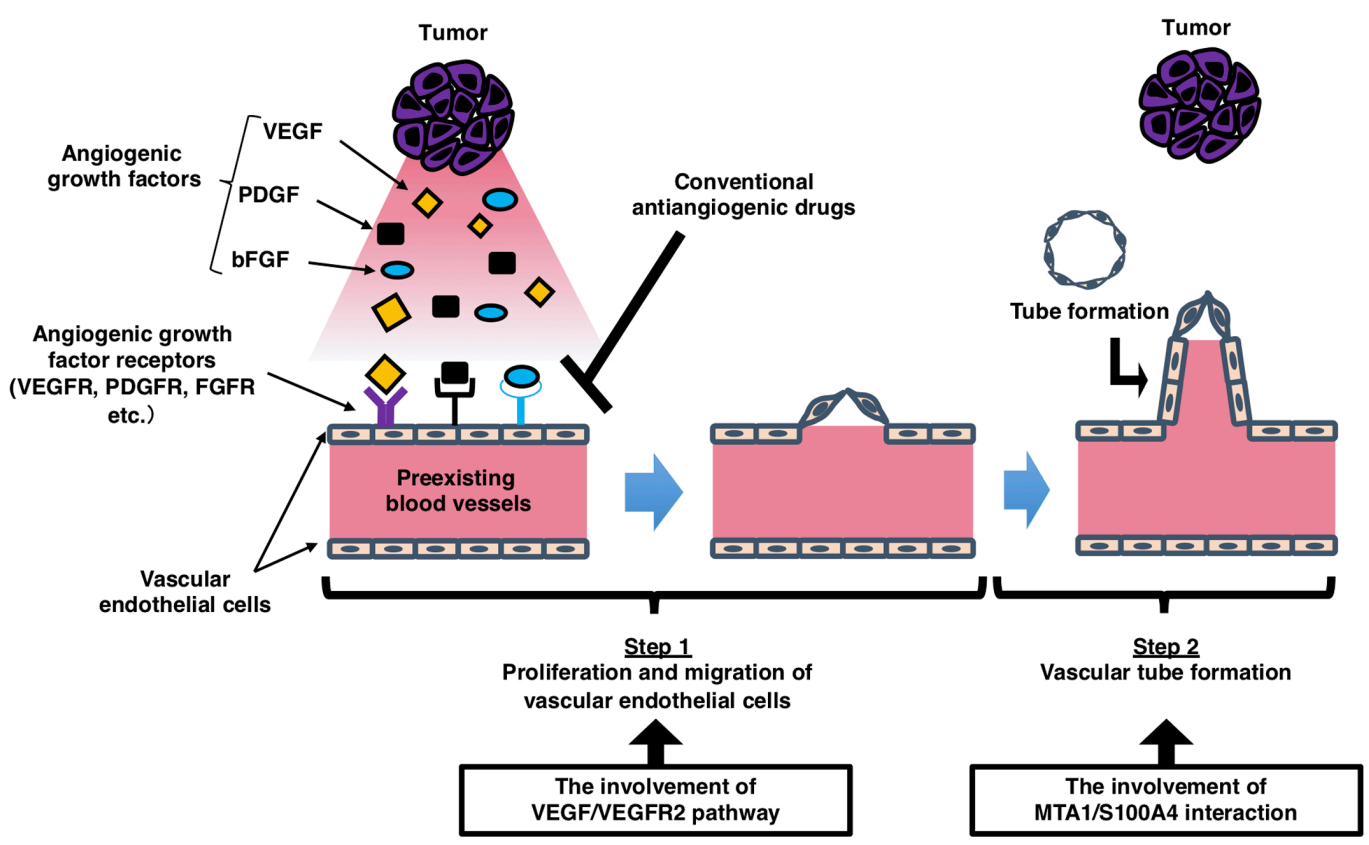

Figure 7. Schematic diagram of the process of tumor angiogenesis. New blood vessels are generated from pre-existing blood vessels through angiogenic growth factors secreted by tumor cells. The vascular endothelial cells in pre-existing blood vessels degrade the basement membrane, proliferate, migrate and form sprouts after the activation of angiogenic stimuli (step 1). Subsequently, these sprouts lead to vascular tube formation, followed by blood vessel maturation to complete the tube structure through which blood can flow (step 2). VEGF stimulates endothelial proliferation and migration in step 1, whereas MTA1 is primarily associated with vascular tube formation in step 2. VEGF, vascular endothelial growth factor; VEGFR, VEGF receptor 2; PDGF, platelet-derived growth factor; bFGF, basic fibroblast growth factor; FGFR, FGF receptor; PDGFR, platelet-derived growth factor receptor; MTA1, metastasis-associated protein 1; S100A4, S100 calcium-binding protein A4. 


\section{Acknowledgements}

The authors would like to thank Dr H Endo (The University of Tokyo, Tokyo, Japan) for kindly providing MSS31 cells and Dr K. Takenaga (Chiba Cancer Center Research Institute, Chiba, Japan) for providing the MTA1 expression vector.

\section{Funding}

No funding was received.

\section{Availability of data and materials}

The datasets used and/or analyzed during the current study are available from the corresponding author on reasonable request.

\section{Authors' contributions}

MI, MO, NU, TO, HK and FO designed the experiments. MI, NU, TO and MO performed the experiments and analyzed the data. MI, MO, NU, TO, HK and FO wrote, reviewed and revised the manuscript. $\mathrm{MI}, \mathrm{MO}$ and NU confirm the authenticity of all the raw data. All authors reviewed and approved the final manuscript.

\section{Ethics approval and consent to participate}

Not applicable.

\section{Patient consent for publication}

Not applicable.

\section{Competing interests}

The authors declare that they have no competing interests.

\section{References}

1. Toh Y, Pencil SD and Nicolson GL: A novel candidate metastasisassociated gene, mtal, differentially expressed in highly metastatic mammary adenocarcinoma cell lines. cDNA cloning, expression, and protein analyses. J Biol Chem 269: 22958-22963, 1994.

2. Toh Y, Kuwano H, Mori M, Nicolson GL and Sugimachi K: Overexpression of metastasis-associated MTA1 mRNA in invasive oesophageal carcinomas. Br J Cancer 79: 1723-1726, 1999.

3. Toh Y, Oki E, Oda S, Tokunaga E, Ohno S, Maehara Y, Nicolson GL and Sugimachi K: Overexpression of the MTA1 gene in gastrointestinal carcinomas: Correlation with invasion and metastasis. Int J Cancer 74: 459-463, 1997.

4. Zhu X, Guo Y, Li X, Ding Y and Chen L: Metastasis-associated protein 1 nuclear expression is associated with tumor progression and clinical outcome in patients with non-small cell lung cancer. J Thorac Oncol 5: 1159-1166, 2010.

5. Jang KS, Paik SS, Chung H, Oh YH and Kong G: MTA1 overexpression correlates significantly with tumor grade and angiogenesis in human breast cancers. Cancer Sci 97: 374-379, 2006.

6. Dannenmann C, Shabani N, Friese K, Jeschke U, Mylonas I and Brüning A: The metastasis-associated gene MTA1 is upregulated in advanced ovarian cancer, represses ERbeta, and enhances expression of oncogenic cytokine GRO. Cancer Biol Ther 7: 1460-1467, 2008.
7. Nicolson GL, Nawa A, Toh Y, Taniguchi S, Nishimori K and Moustafa A: Tumor metastasis-associated human MTA1 gene and its MTA1 protein product: Role in epithelial cancer cell invasion, proliferation and nuclear regulation. Clin Exp Metastasis 20: 19-24, 2003.

8. Kai L, Wang J, Ivanovic M, Chung YT, Laskin WB, Schulze-Hoepfner F, Mirochnik Y, Satcher RL Jr and Levenson AS: Targeting prostate cancer angiogenesis through metastasis-associated protein 1 (MTA1). Prostate 71: 268-280, 2011.

9. Pakala SB, Rayala SK, Wang RA, Ohshiro K, Mudvari P, Reddy SD, Zheng Y, Pires R, Casimiro S, Pillai MR, et al: MTA1 promotes STAT3 transcription and pulmonary metastasis in breast cancer. Cancer Res 73: 3761-3770, 2013.

10. Ishikawa M, Osaki M, Yamagishi M, Onuma K, Ito H, Okada F and Endo H: Correlation of two distinct metastasis-associated proteins, MTA1 and S100A4, in angiogenesis for promoting tumor growth. Oncogene 38: 4715-4728, 2019.

11. Ran FA, Hsu PD, Wright J, Agarwala V, Scott DA and Zhang F: Genome engineering using the CRISPR-Cas9 system. Nat Protoc 8: 2281-2308, 2013.

12. Livak KJ and Schmittgen TD: Analysis of relative gene expression data using real-time quantitative PCR and the 2(-Delta Delta C(T)) method. Methods 25: 402-408, 2001.

13. Welti J, Loges S, Dimmeler S and Carmeliet P: Recent molecular discoveries in angiogenesis and antiangiogenic therapies in cancer. J Clin Invest 123: 3190-3200, 2013.

14. Teleanu RI, Chircov C, Grumezescu AM and Teleanu DM: Tumor Angiogenesis and Anti-Angiogenic Strategies for Cancer Treatment. J Clin Med 9: E84, 2019.

15. Jászai J and Schmidt MH: Trends and challenges in tumor anti-angiogenic therapies. Cells 8: E1102, 2019.

16. Rajabi M and Mousa SA: The role of angiogenesis in cancer treatment. Biomedicines 5: E34, 2017.

17. Herbert SP and Stainier DY: Molecular control of endothelial cell behaviour during blood vessel morphogenesis. Nat Rev Mol Cell Biol 12: 551-564, 2011.

18. Ferrara N, Gerber HP and LeCouter J: The biology of VEGF and its receptors. Nat Med 9: 669-676, 2003.

19. Apte RS, Chen DS and Ferrara N: VEGF in signaling and disease: Beyond discovery and development. Cell 176: 1248-1264, 2019.

20. Folkman J: Tumor angiogenesis: Therapeutic implications. N Engl J Med 285: 1182-1186, 1971.

21. Jayson GC, Kerbel R, Ellis LM and Harris AL: Antiangiogenic therapy in oncology: Current status and future directions. Lancet 388: 518-529, 2016.

22. Ferrara $\mathrm{N}$ and Kerbel RS: Angiogenesis as a therapeutic target. Nature 438: 967-974, 2005.

23. Haibe Y, Kreidieh M,El Hajj H, Khalifeh I, Mukherji D, Temraz S and Shamseddine A: Resistance mechanisms to anti-angiogenic therapies in cancer. Front Oncol 10: 221, 2020.

24. Bergers $G$ and Hanahan D: Modes of resistance to anti-angiogenic therapy. Nat Rev Cancer 8: 592-603, 2008.

25. van Beijnum JR, Nowak-Sliwinska P, Huijbers EJ, Thijssen VL and Griffioen AW: The great escape; the hallmarks of resistance to antiangiogenic therapy. Pharmacol Rev 67: 441-461, 2015.

26. Pakala SB, Reddy SD, Bui-Nguyen TM, Rangparia SS, Bommana A and Kumar R: MTA1 coregulator regulates LPS response via MyD88-dependent signaling. J Biol Chem 285: 32787-32792, 2010.

27. Li DQ, Pakala SB, Reddy SD, Peng S, Balasenthil S, Deng CX, Lee CC, Rea MA and Kumar R: Metastasis-associated protein 1 is an integral component of the circadian molecular machinery. Nat Commun 4: 2545, 2013.

28. Mitchell DC and Bryan BA: Anti-angiogenic therapy: Adapting strategies to overcome resistant tumors. J Cell Biochem 111: $543-553,2010$.

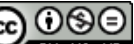

This work is licensed under a Creative Commons Attribution-NonCommercial-NoDerivatives 4.0 International (CC BY-NC-ND 4.0) License. 